\title{
Calcimimetic restores diabetic peripheral neuropathy by ameliorating apoptosis and improving autophagy
}

\author{
You Chul Chung ${ }^{1}$, Ji Hee Lim², ${ }^{2,3}$ Hyun Mi Oh', Hyung Wook Kim², Min Young Kim², ${ }^{2}$ Eun Nim Kim², Yaeni Kim², \\ Yoon Sik Chang ${ }^{2}$, Hye Won Kim ${ }^{1}$ and Cheol Whee Park ${ }^{2,3}$
}

\begin{abstract}
Decreased AMPK-eNOS bioavailability mediates the development of diabetic peripheral neuropathy (DPN) through increased apoptosis and decreased autophagy activity in relation to oxidative stress. Schwann cells are responsible for maintaining structural and functional integrity of neurons and for repairing damaged nerves. We evaluated the neuroprotective effect of cinacalcet on DPN by activating the AMPK-eNOS pathway using $d b / d b$ mice and human Schwann cells (HSCs). Sciatic nerve of $d b / d b$ mice was characterized by disorganized myelin, axonal shrinkage, and degeneration that were accompanied by marked fibrosis, inflammation, and apoptosis. These phenotypical alterations were significantly improved by cinacalcet treatment along with improvement in sensorimotor functional parameters. Cinacalcet demonstrated favorable effects through increased expression and activation of calcium-sensing receptor (CaSR)-CaMKKß and phosphorylation of AMPK-eNOS signaling in diabetic sciatic nerve. Cinacalcet decreased apoptosis and increased autophagy activity in relation to decreased oxidative stress in HSCs cultured in high-glucose medium as well. This was accompanied by increased expression of the CaSR, intracellular $\mathrm{Ca}^{++}\left(\left[\mathrm{Ca}^{++}\right] \mathrm{i}\right)$ levels, and CaMKKR-LKB1AMPK signaling pathway, resulting in the net effect of increased eNOS phosphorylation, NOx concentration, Bcl-2/Bax ratio, beclin 1, and LC3-II/LC3-I ratio. These results demonstrated that cinacalcet treatment ameliorates inflammation, apoptosis, and autophagy through increased expression of the $\mathrm{CaSR},\left[\mathrm{Ca}^{++}\right]$i levels and subsequent activation of CaMKKß-LKB-1-AMPK-eNOS pathway in the sciatic nerve and HSCs under diabetic condition. Therefore, cinacalcet may play an important role in the restoration and amelioration of DPN by ameliorating apoptosis and improving autophagy.
\end{abstract}

\section{Introduction}

Diabetic peripheral neuropathy (DPN) is one of the most common complications of diabetes in $>50-60 \%$ of all diabetic patients, and it is also the leading cause of amputation worldwide ${ }^{1,2}$. The early changes in patients with DPN include accumulation of extracellular matrix

\footnotetext{
Correspondence: Hye Won Kim (kimhw@catholic.ac.kr) or

Cheol Whee Park (cheolwhee@hanmail.net)

${ }^{1}$ Department of Rehabilitation Medicine, College of Medicine, The Catholic

University of Korea, Seoul, Korea

2Division of Nephrology, Department of Internal Medicine, College of

Medicine, The Catholic University of Korea, Seoul, Korea

Full list of author information is available at the end of the article.

Edited by G.M. Fimia
}

proteins, inflammation, axonal degeneration, and loss of unmylelinated fibers, which cause sensorimotor conduction delays and irreversible nerve damage. It is well known that hyperglycemia plays a main role in $\mathrm{DPN}^{3-7}$ with regard to the changes in oxidative-nitrosative stress, neuro-inflammation, mitochondrial dysfunction, bioenergetic crisis, and demyelination ${ }^{7}$.

Schwann cells (SCs) are specialized glial cells in the peripheral nervous system that are responsible for maintaining structural and functional integrity of neurons and for repairing damaged nerves ${ }^{8,9}$. Hyperglycemia-induced SC damages may reduce nerve conduction velocity, accelerate axonal atrophy, and impair axonal regeneration ${ }^{10}$.

\section{(c) The Author(s) 2018}

(c) (i) Open Access This article is licensed under a Creative Commons Attribution 4.0 International License, which permits use, sharing, adaptation, distribution and reproduction cc) in any medium or format, as long as you give appropriate credit to the original author(s) and the source, provide a link to the Creative Commons license, and indicate if changes were made. The images or other third party material in this article are included in the article's Creative Commons license, unless indicated otherwise in a credit line to the material. If material is not included in the article's Creative Commons license and your intended use is not permitted by statutory regulation or exceeds the permitted use, you will need to obtain permission directly from the copyright holder. To view a copy of this license, visit http://creativecommons.org/licenses/by/4.0/. 
Moreover, hyperglycemia-induced SC damages include such morphological changes as swelling and vacuolization that result in the destruction of organelles. Clearance of defective organelles constitutes the very core of the autophagy process that is an important physiological and defensive mechanism of the cell and body under such deranged metabolic conditions as nutrient or energy excess and deprivation ${ }^{11}$. Chronic hyperglycemia with diabetes impairs cellular autophagy and exacerbates apoptosis associated with $\mathrm{DPN}^{7,12}$. Autophagy promotes cell survival by sequestering senescent or damaged organelles/proteins in autophagosomes for recycling of their products ${ }^{11}$. Therefore, an enhancement of autophagy and a concomitant suppression of apoptosis of SCs might be the optimal strategy for the prevention and regression of DPN.

AMP-activated protein kinase (AMPK) is a master controller of cellular energy balance that activates catabolic pathways in state of energy deprivation ${ }^{13}$. Chronic nutrient excess state associated with prolonged diabetes triggers a switching off of AMPK, which results in impaired peroxisome proliferator-activated receptor $\gamma$ coactivator-1 $\alpha$ (PGC-1 $\alpha)$ activity and diminished mitochondrial $^{14}$ and endothelial nitric oxide synthase (eNOS) activities ${ }^{15}$, leading to neurodegeneration in patients with DPN. The important mode of AMPK activation relies on phosphorylation at the 172nd threonine residue of the $\alpha$ subunit by upstream kinases, including $\mathrm{Ca}^{++} /$calmodulindependent protein kinase kinase $\beta(\mathrm{CaMKK} \beta)$ and liver kinase B1 (LKB1). The LKB1 forms a complex with STRAD and MO25 in response to an elevation in AMP/ ATP ratio ${ }^{16}$, which phosphorylates the AMPK $\alpha$ subunit to trigger the AMPK pathway. CaMKK $\beta$ is an alternative upstream kinase of AMPK that responds to the change in intracellular $\mathrm{Ca}^{++}\left(\left[\mathrm{Ca}^{++}\right] \mathrm{i}\right)$ concentration. Elevated $\left[\mathrm{Ca}^{++}\right] \mathrm{i}$ increases the activity of AMPK, independent of the adenylate energy balance ${ }^{17}$.

The calcimimetic, (R)-N-(3-(3-(trifluoromethyl)phenyl) propyl)-1-(1-napthyl)ethylamine hydrochloride (cinacalcet), devised originally for the treatment of secondary hyperparathyroidism, exerts its effect by stimulating $\mathrm{Ca}^{++}$-sensing receptor (CaSR) mainly in the parathyroid glands ${ }^{18}$. Activated upon $\mathrm{Ca}^{++}$ions, the expression of cellular surface CaSR is crucial for maintaining a stable serum $\mathrm{Ca}^{++}$, which is achieved primarily through the regulation of parathyroid hormone secretion and renal $\mathrm{Ca}^{++}$excretion. Interestingly, the expression of CaSR has been demonstrated in the vasculature and perivascular sensory nerves ${ }^{18,19}$. CaSR activation by cinacalcet is known to activate CaMMK-LKB1-AMPK pathway. Activation of AMPK and LKB-1 is crucial for SC-mediated axonal maintenance while LKB deletion is responsible for axonal degeneration ${ }^{20}$. Moreover, the CaSR is known to modulate cell proliferation and apoptosis and coordinate oncogene expression, chemotaxis, and autophagy.
Exposed under constant metabolic stress, these axons and $\mathrm{SCs}$ are prone to mitochondrial dysfunction featuring derangements in $\left[\mathrm{Ca}^{++}\right] \mathrm{i}$ homeostasis and associated downstream signaling that are key causal factors for the development of DPN, making it an ideal therapeutic target at the same time ${ }^{21}$.

To date, there is no curative therapy currently available to deter the progression of DPN; only a handful of studies have demonstrated improvements in indices of neuropathy through the activation of AMPK in cultured neurons $^{22}$ and peripheral nerves of type 1 diabetic rats ${ }^{23}$. On this account, we assumed that cinacalcet treatment may modulate DPN activity through the axis delineated in the previous study. Thus we investigated the protective and/ or reversal effect of cinacalcet against neural glucotoxicity through the changes in the AMPK-eNOS pathway in the sciatic nerve of $d b / d b$ mice and human Schwann cells (HSCs).

\section{Materials and methods \\ Experimental animals and assessment of peripheral nerve function}

All animal experiments were performed in accordance with the Laboratory Animals Welfare Act, the Guide for the Care and Use of Laboratory Animals, and were approved by the Institutional Animal Care and Use Committee (IACUC) at College of Medicine, the Catholic University of Korea (CUMC-2014- 0165-01). Eight-week-old male C57BLKS/J $d b / m$ and $d b / d b$ mice were purchased from Jackson Laboratories (Bar Harbor, ME, USA) and $d b / m$ and $d b / d b$ mice were divided into four groups. Cinacalcet $(10 \mathrm{mg} / \mathrm{kg})$ mixed into standard chow diet or a regular diet was administered to $d b / d b$ mice $(d b / d b+$ cina; $n=8)$ and ageand gender-matched $d b / m$ mice $(d b / \mathrm{m}+$ cina; $\mathrm{n}=8)$ for 12 weeks starting at 8 weeks of age.

After 12 weeks of cinacalcet treatment, we performed electrophysiological and sensory threshold tests in the following order: tactile responses (a response $50 \%$ of the times the tip is applied to the hind paw) to stimulation using flexible von Frey filaments and then sciatic motor nerve conduction latency (MNCL), as described previously $^{15}$. After the tests were completed, blood was collected from the left ventricle and the plasma was stored at $-70{ }^{\circ} \mathrm{C}$ for subsequent analyses, and we collected the sciatic nerves under general anesthesia with $10 \mathrm{mg} / \mathrm{kg}$ xylazine hydrochloride (Rompun; Bayer, Leuverkusen, Germany) and $30 \mathrm{mg} / \mathrm{kg}$ tiletamine plus zolazepam (Zoletil; Virbac, Carros, France). Some sciatic nerve samples were fixed in normal buffered $4 \%$ formalin for immunohistochemistry, and the others were stored in a solution for electron microscopy. We also collected the sciatic nerves in 8-week old male $d b / m$ and $d b / d b$ mice for evaluation of cinacalcet effect on the recovery of DPN. 


\section{Assessment of blood glucose, $\mathrm{HbA1c}$, plasma ionized calcium, and $\mathrm{PO}_{4}{ }^{-}$concentrations}

After 12 weeks of treatment with cinacalcet, blood glucose was measured using an Accucheck meter (Roche Diagnostics, St. Louis, MO). Hemoglobin A1c (HbA1c) was determined on red cell lysates using highperformance liquid chromatography (BioRad, Hercules, CA). Plasma ionized calcium $\left(\mathrm{iCa}^{++}\right)$and $\mathrm{PO}_{4}{ }^{-}$concentrations were measured using colorimetry (Samkwang Medical Laboratory, Seoul, Korea).

\section{Light and electron microscopic analysis Nerve morphology}

Sciatic nerve samples were fixed in $4 \%$ paraformaldehyde. Trichrome-stained nerves were used to examine the effect of cinacalcet on nerve fibrosis. Ten consecutive nerve cross-sections were photographed using a digital camera (Olympus DP11; Olympus America, Melville, NY) by an examiner who was blinded to the tissue source. Each nerve section was sampled in a serpentine pattern such that the entire nerve section was analyzed with no overlapping fields. We performed immunohistochemistry for type IV collagen (Col IV; Biodesign International, Saco, ME, USA) and 8-hydroxy-deoxyguanosine (8-OHdG; JalCA, Fukuroi, Shizuoka, Japan), an oxidative DNA damage marker.

Immunofluorescence double staining for $F 4 / 80$ and TdTmediated dUTP-biotin nick end labeling (TUNEL) and SOX10, $\beta 3$-tubulin, and LC3

For immunofluorescence double staining, apoptosis was detected by the ApopTag Fluorescein In Situ Apoptosis Detection Kit (S7110; Chemicon International, Temecula, CA), as described previously ${ }^{15}$. And then, sections were incubated overnight with cell surface glycoprotein F4/80 (Serotec, Oxford, UK) and a Texas red-labeled secondary antibody and counterstained with 4,6-diamidino-2-phenylindole (DAPI). We also performed immunofluorescence double staining for SOX10 (Abcam, Cambridge, UK), $\beta 3$-tubulin (1:50; Cell Signaling Technology, Danvers, MA), and LC3 (1:200; Sigma-Aldrich, St. Louis, MO, USA). The fluorescent images were examined under a laser scanning confocal microscope system (Carl Zeiss LSM 700, Oberkochen, Germany).

\section{Electron microscopy}

For transmission electron microscopy (TEM), sciatic nerves specimens were fixed in $4 \%$ paraformaldehyde and $2.5 \%$ glutaraldehyde in $0.1 \mathrm{M}$ phosphate buffer overnight at $4{ }^{\circ} \mathrm{C}$. After washing in $0.1 \mathrm{M}$ phosphate buffer, the specimens were post-fixed with $1 \%$ osmium tetroxide in the same buffer for $1 \mathrm{~h}$. The specimens were then dehydrated using a series of graded ethanol, exchanged through acetone, and embedded in Epon 812. Ultrathin sections $(70-80 \mathrm{~nm})$ were obtained by ultramicrotome (Leica Ultracut UCT, Leica, Germany) and were double stained with uranyl acetate and lead citrate and examined in transmission electron microscope (JEM 1010, Tokyo, Japan) at $60 \mathrm{kV}$. We measured areas of unmyelinated fiber, axonal diameters, $G$ ratio, and the number of degenerative fiber using NIH Image J.

\section{Western blot analysis}

The total proteins of the sciatic nerve tissues were extracted with a Pro-Prep Protein Extraction Solution (Intron Biotechnology, Gyeonggi-Do, Korea), following the manufacturer's instructions. Western blot assay was performed with specific antibodies for CaSR (Thermo Fisher Scientific Inc, Waltham, MA, USA), CaMKK $\beta$ (Santa Cruz Biotechnology, Santa Cruz, CA, USA), total LKB1 (Cell Signaling Technology, Danvers, MA, USA), phosphor-Ser ${ }^{428}$ LKB1 (Cell Signaling Technology, Danvers, MA, USA), total AMPK (Cell Signaling Technology, Danvers, MA, USA), phospho-Thr ${ }^{172}$ AMPK (1:2000; Cell Signaling Technology, Danvers, MA, USA), total eNOS (Cell Signaling Technology, Danvers, MA, USA), phospho-Ser ${ }^{1177}$ eNOS (Cell Signaling Technology, Danvers, MA, USA), PGC- $1 \alpha$ (Novus Biologicals, Littleton, CO, USA), B cell leukemia/lymphoma 2 (Bcl-2) (Santa Cruz Biotechnology, Santa Cruz, CA, USA), BCL-2associated X protein (Bax) (Santa Cruz Biotechnology, Santa Cruz, CA, USA), beclin-1 (Novus Biologicals, Littleton, CO), and LC-3 (Sigma-Aldrich, St. Louis, MO). After incubation with horseradish peroxidase-conjugated anti-mouse or anti-rabbit IgG (Cell Signaling Technology, Danvers, MA), target proteins were visualized by an enhanced chemiluminescence substrate (ECL Plus, GE Healthcare Bio-Sciences, Piscataway, NJ).

\section{HSC culture study \\ $\left[\mathrm{Ca}^{++}\right] \mathrm{i}$ measurement}

HSCs were cultured in SC Medium (ScienCell Research Laboratories, San Diego, CA), as described previously ${ }^{15}$. Passages $4-8$ were used in all experiments. The HSCs were exposed to low glucose $(5 \mathrm{mmol} / \mathrm{L} \mathrm{D}$-glucose; lowglucose) or high glucose $(40 \mathrm{mmol} / \mathrm{L} \mathrm{D}$-glucose), with or without the additional 24-h application of cinacalcet (15 and $100 \mathrm{nM}$ ). Calcium concentrations were determined based on the ratio of fura- 2 fluorescence intensities at $340-\mathrm{nm}$ excitation and $380-\mathrm{nm}$ excitation. The ratio of $340 / 380$ directly reflects the amount of $\left[\mathrm{Ca}^{++}\right]$i. The $340-$ $\mathrm{nm}$ fluorescence of fura- 2 increases and the $380-\mathrm{nm}$ of fura-2 decreases with increasing $\left[\mathrm{Ca}^{++}\right]$i. For $\left[\mathrm{Ca}^{++}\right] \mathrm{i}$ measurements, HSCs $(20,000$ cells/well) were plated on black 96-well plates with a clear bottom in complete medium. After 1 day, the cultures were serum-starved for $2 \mathrm{~h}$ in SC media. In the last $45 \mathrm{~min}$ of serum starvation, $5 \mathrm{mM}$ FURA-2AM without $\mathrm{Ca}^{++}$was added to the cells 
and then rinsed with Hank's Balanced Salt Solution (GibcoBRL, Grand Island, NY). FURA-2AM-loaded cells were sequentially excited at 340 and $380 \mathrm{~nm}$ by spectrophotometer microplate reader (Synergy MX; BioTek, Winooski, VT).

Immunofluorescence and western blot analyses in the HSCS

To evaluate the effects of cinacalcet on CsSR, CaMKK $\beta$, phospho-Ser ${ }^{428}$ LKB1, and phospho-Thr ${ }^{172}$ AMPK expression, we performed immunofluorescence analysis with specific antibodies for CaSR, CaMKK $\beta$, phospho-Ser ${ }^{428}$ LKB1, and phospho-Thr ${ }^{172}$ AMPK by using tyramide signal amplification fluorescence system and counterstained with DAPI. In addition, the total proteins of the HSCs were extracted with a Pro-Prep Protein Extraction Solution, following the manufacturer's instructions. After incubation with horseradish peroxidase-conjugated anti-mouse or anti-rabbit IgG (Cell Signaling Technology, Danvers, MA), target proteins were visualized by an enhanced chemiluminescence substrate (ECL Plus, GE, Healthcare Bio-Science, Piscataway, $\mathrm{NJ})$. To evaluate the anti-apoptotic effects of cinacalcet on HSCs in high-glucose medium, the number of TUNELpositive cells was counted in 10 randomly chosen fields at a magnification of $\times 400$. We also measured the concentration of NOx to quantify $\mathrm{NO}$ production in cell-culture media. The total $\mathrm{NO}_{3}$ and $\mathrm{NO}_{2}$ were quantified using the Nitric Oxide Assay Kit (Bio Vision, Mountain View, CA).

\section{HSCs with small interfering RNA (siRNA) transfection}

siRNAs, targeted against CaMKK $\beta$, LKB1, and scrambled siRNA (siRNA cont), were complexed with the transfection reagent (Lipofectamine 2000; Invitrogen, Carlsbad, CA), according to the manufacturer's instructions. The sequences of the siRNAs are as follows: CaMKK $\beta, \quad$ 5'-GGAUCUGAUCAAAGGCAUCTT-3'; LKB1, 5'-GGACUGACGUGUAGAACAATT-3'; and nonspecific scrambled siRNA, 5'-CCUACGCCACCA AUUUCGU-3' (Bioneer, Daejeon, Korea). HSCs in 6-well plates were transfected with a final concentration of $50 \mathrm{nM}$ CaMKK $\beta$ and LKB1 siRNAs for $24 \mathrm{~h}$ using the transfection reagent (Lipofectamine 2000) in Opti-MEM media (Gibco Life Technologies), according to the manufacturer's instructions. After transfection, cells were treated with cinacalcet $(5 \mathrm{nM})$ in high-glucose medium to evaluate the effects of siRNAs on HSC reactions.

\section{Data analysis}

SPSS version 16 (SPSS. Inc., Chicago, IL) was used to conduct the statistical analysis. Group differences were evaluated using an analysis of variance with the Bonferroni correction. Non-normally distributed data were analyzed by Mann-Whitney $U$ test. The results are expressed as mean $\pm \mathrm{SD}$. A $P$ value of $<0.05$ was considered statistically significant.

\section{Results}

Body weight, blood $\mathrm{HbA} 1 \mathrm{c}$, glucose, $\mathrm{iCa}^{++}$, and $\mathrm{PO}_{4}{ }^{3-}$ levels

The body weights of $d b / d b$ mice were greater than those of $\mathrm{db} / \mathrm{m}$ mice in both the cinacalcet treatment and control groups at the end of the study $(p<0.001$, Table 1$)$. No changes in body weight were noted in $\mathrm{db} / \mathrm{m}$ and $\mathrm{db} / \mathrm{db}$ mice following the 12-week treatment with cinacalcet. HbA1c and fasting blood sugar concentrations were significantly higher in $d b / d b$ mice than those in $d b / m$ mice in both the cinacalcet treatment and control groups $(p<0.001$, Table 1$)$. Interestingly, cinacalcet treatment did not change fasting blood glucose, HbA1c, or serum $\mathrm{iCa}^{++}$and $\mathrm{PO}_{4}{ }^{3-}$ concentrations in $d b / d b$ or $d b / m$ mice.

\section{Assessment of peripheral nerve function}

Tactile response thresholds, motor nerve conduction latency, and action potential amplitude in the sciatic nerve

Tactile response thresholds (Fig. 1a, $1.09 \pm 0.28$ vs. $0.0 .53 \pm 0.23 \mathrm{~g}, p<0.001$ ) and MNCL (Fig. 1b, $1.81 \pm 0.06$ vs. $1.51 \pm 0.05 \mathrm{~ms}, p<0.01)$ were increased in $d b / d b$ cont compared with those in $d b / m$ cont mice at the end of the 12-week study. Interestingly, cinacalcet treatment significantly improved the tactile response threshold and MNCL in $d b / d b$ mice compared to those in $d b / d b$ control (Fig. 1a, $0.74 \pm 0.18$ vs. $0.36 \pm 0.14 \mathrm{~g}, p<0.01$ and Fig. $1 \mathrm{~b}$, $1.64 \pm 0.05$, vs. $1.48 \pm 0.06 \mathrm{~ms}, p<0.01$, respectively) to the levels similar to those in $\mathrm{db} / \mathrm{m}$ mice. Consistent with the improvement in the tactile response threshold and MNCL, cinacalcet treatment increased action potential amplitude in $d b / d b$ mice (Fig. 1c, $873 \pm 224$ vs. $1199 \pm$ $124 \mu \mathrm{m}, p<0.01)$. However, there were no changes in the tactile response, sciatic motor conduction latency, and action potential amplitude in the $d b / m$ mice treated with or without cinacalcet.

Table 1 Biochemical and physical characteristics of all the study groups

\begin{tabular}{lllll}
\hline & $\boldsymbol{d} \boldsymbol{b} / \boldsymbol{m}$ cont & $\begin{array}{l}\boldsymbol{d} \boldsymbol{b} / \boldsymbol{m} \\
+ \text { cina }\end{array}$ & $\boldsymbol{d} \boldsymbol{b} / \boldsymbol{d} \boldsymbol{b}$ cont & $\begin{array}{l}\boldsymbol{d} \boldsymbol{b} / \boldsymbol{d} \boldsymbol{b} \\
+ \text { cina }\end{array}$ \\
\hline Body wt (g) & $31.9 \pm 1.7$ & $31.3 \pm 2.0$ & $55.7 \pm 5.5^{\mathrm{a}}$ & $54.5 \pm 7.4^{\mathrm{a}}$ \\
$\mathrm{HbA1c}(\%)$ & $4.5 \pm 0.3$ & $4.5 \pm 0.4$ & $12.1 \pm 1.3^{\mathrm{a}}$ & $11.8 \pm 1.4^{\mathrm{a}}$ \\
$\mathrm{HbA1c}(\mathrm{mmol} /$ & $(26 \pm 0.8)$ & $(26 \pm 0.9)$ & $\left(109 \pm 3.7^{\mathrm{a}}\right)$ & $\left(105 \pm 3.9^{\mathrm{a}}\right)$ \\
mol) & & & & \\
$\mathrm{Glucose}(\mathrm{mg} / \mathrm{dl})$ & $211 \pm 25$ & $202 \pm 20$ & $554 \pm 39^{\mathrm{a}}$ & $544 \pm 54^{\mathrm{a}}$ \\
$\mathrm{iCa}^{++}(\mathrm{mmol} / \mathrm{l})$ & $1.29 \pm 0.03$ & $1.24 \pm 0.05$ & $1.33 \pm 0.06$ & $1.29 \pm 0.07$ \\
$\mathrm{PO}_{4}{ }^{3-}(\mathrm{mmol} / \mathrm{l})$ & $3.8 \pm 0.8$ & $3.9 \pm 0.9$ & $4.0 \pm 1.0$ & $3.9 \pm 0.8$ \\
\hline
\end{tabular}

$\mathrm{Ca}$ total calcium, $\mathrm{Cr}$ creatinine, $\mathrm{iCa}^{++}$serum ionized $\mathrm{Ca}^{++}$

${ }^{\text {a }} P<0.001$ compared to the other groups ( $n=8$ in each experiment) 
A

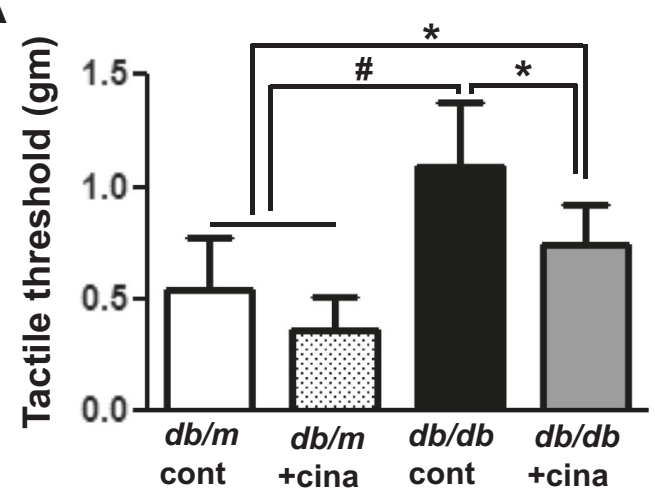

C

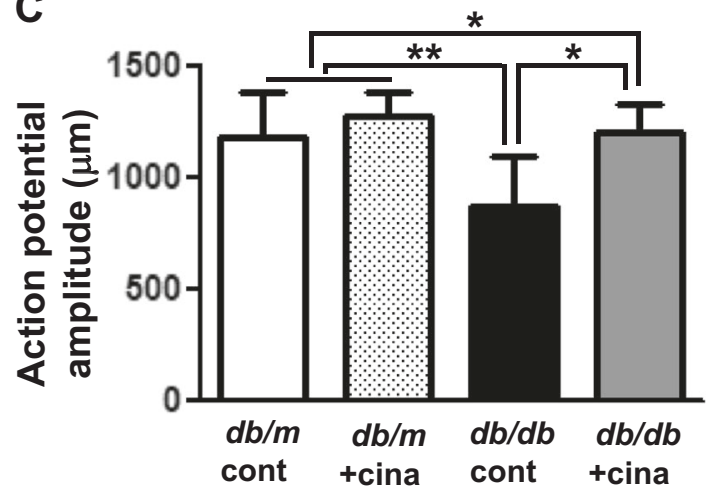

B

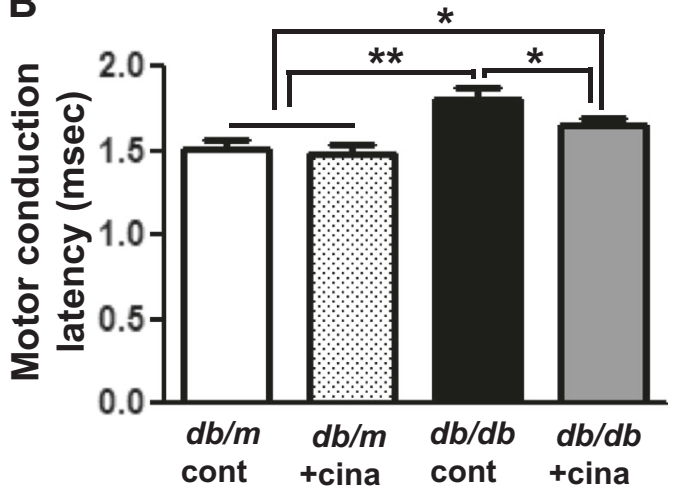

Fig. 1 Cinacalcet improves sensory and motor functions of the sciatic nerve in $\boldsymbol{d} b / \mathbf{d} b$ mice. $\mathbf{a}-\mathbf{c}$ Effects of cinacalcet on the tactile threshold (a), motor conduction latency (b), and action potential amplitude (c) were determined at 20 weeks in $\mathrm{db} / \mathrm{m}$ and $\mathrm{db} / \mathrm{db}$ mice with or without cinacalcet treatment. ( $n=8$ in each groups) ${ }^{*} p<0.05,{ }^{* *} p<0.01$, and ${ }^{*} p<0.001$ compared with the other groups

\section{Assessment of nerve pathology}

\section{Expressions of nerve fibrosis and Collagen IV (Col IV)}

The sciatic nerve in the $d b / d b$ cont mice showed significant nerve fibrosis as reflected by increased trichromestained area when compared with that of $d b / m$ mice (Fig. 2a, b, $8.3 \pm 2.1$ vs. $3.4 \pm 1.6 \%, p<0.01$ ) Immunohistochemical staining revealed increased expression of Col IV in the sciatic nerve of $d b / d b$ cont compared to that of $\mathrm{db} / \mathrm{m}$ cont mice (Fig. $2 \mathrm{a}, \mathrm{b}, 2.4 \pm 0.5$ vs. $1.0 \pm 0.5$ folds, $p<$ $0.01)$. Increased expression of $\mathrm{Col}$ IV and consistent increase in the extent of fibrotic area in the sciatic nerve of $d b / d b$ control mice were improved to the levels similar to those of $d b / m$ mice with cinacalcet treatment (Fig. 2a, b, $3.8 \pm 1.7 \%$ and $1.2 \pm 0.4$ folds, $p<0.01$, respectively). Thus a 12-week cinacalcet treatment significantly improved fibrosis in the sciatic nerve of $d b / d b$ mice.

\section{8-OH-dG, F4/80-positive cells, and TUNEL-positive cells in the sciatic nerve}

The presence of 8-OH-dG-positive area was more prominent in $d b / d b$ cont mice compared with that in $d b /$ $m$ cont mice, reflecting increased amount of neuronal oxidative stress (Fig. 2a). However, cinacalcet treatment decreased the production of 8-OH-dG to the level comparable to that of $d b / m$ mice (Fig. $2 \mathrm{~b}$ ). We observed the number of TUNEL-positive neural cells, including SC and peripheral neuronal cells which are marked with SOX10 and $\beta 3$-tubulin, respectively. Expression of TUNELpositive cells was significantly increased in $d b / d b$ cont compared with that in $d b / m$ cont mice (Fig. 2c, d, $25 \pm 6$ vs. $13 \pm 3$ cells/high-power field (HPF), $p<0.01)$. Cinacalcet treatment reduced the number of TUNEL-positive neural cells in $d b / d b$ mice $(12 \pm 1.7$ cells/HPF). Additionally, F4/80-positive inflammatory cell infiltration was severe in $d b / d b$ mice when compared with that in nondiabetic $d b / m$ mice $(2.1 \pm 0.5$ vs. $0.2 \pm 0.4$ cells $/ \mathrm{HPF}, p<$ $0.01)$. Cinacalcet treatment reduced the number of F4/80positive cells in $d b / d b$ mice $(0.2 \pm 0.3$ cells/HPF $)$. There were no significant changes in the expression of TUNEL- and F4/80-positive cells in the sciatic nerve of non-diabetic mice with or without cinacalcet treatment. Significant decrease in the expression of LC3 in SOX10and $\beta 3$-tubulin-positive cells in diabetic control mice increased with cinacalcet treatment to the levels 
comparable to those of non-diabetic mice, reflecting the recovery of autophagy process (Fig. 2c, d).

\section{Electron microscopy}

The ultrastructural features of sciatic nerves in $d b / d b$ mice were characterized by severe myelin disruption with axonal shrinkage, degenerated SCs, and decreased amount of unmyelinated fibers with vacuolization when compared with those in $d b / m$ cont mice (Fig. 3a). Sciatic nerves from the $d b / d b$ mice treated with cinacalcet displayed ultra-micro structures that resembled those from $d b / m$ control mice. Significant decreases in both axonal diameter and area and unmyelinated area of $d b / d b$ mice were increased with cinacalcet treatment to the levels comparable to those of non-diabetic mice groups $(p<0.05$ and $p<0.01$, respectively; Fig. $3 \mathrm{~b})$. In contrast, increased $G$ ratio in $d b / d b$ mice was decreased with cinacalcet treatment, indicating optimized axonal myelination ${ }^{24}$ with improved functional and structural indices $(p<0.01$; Fig. 3b).

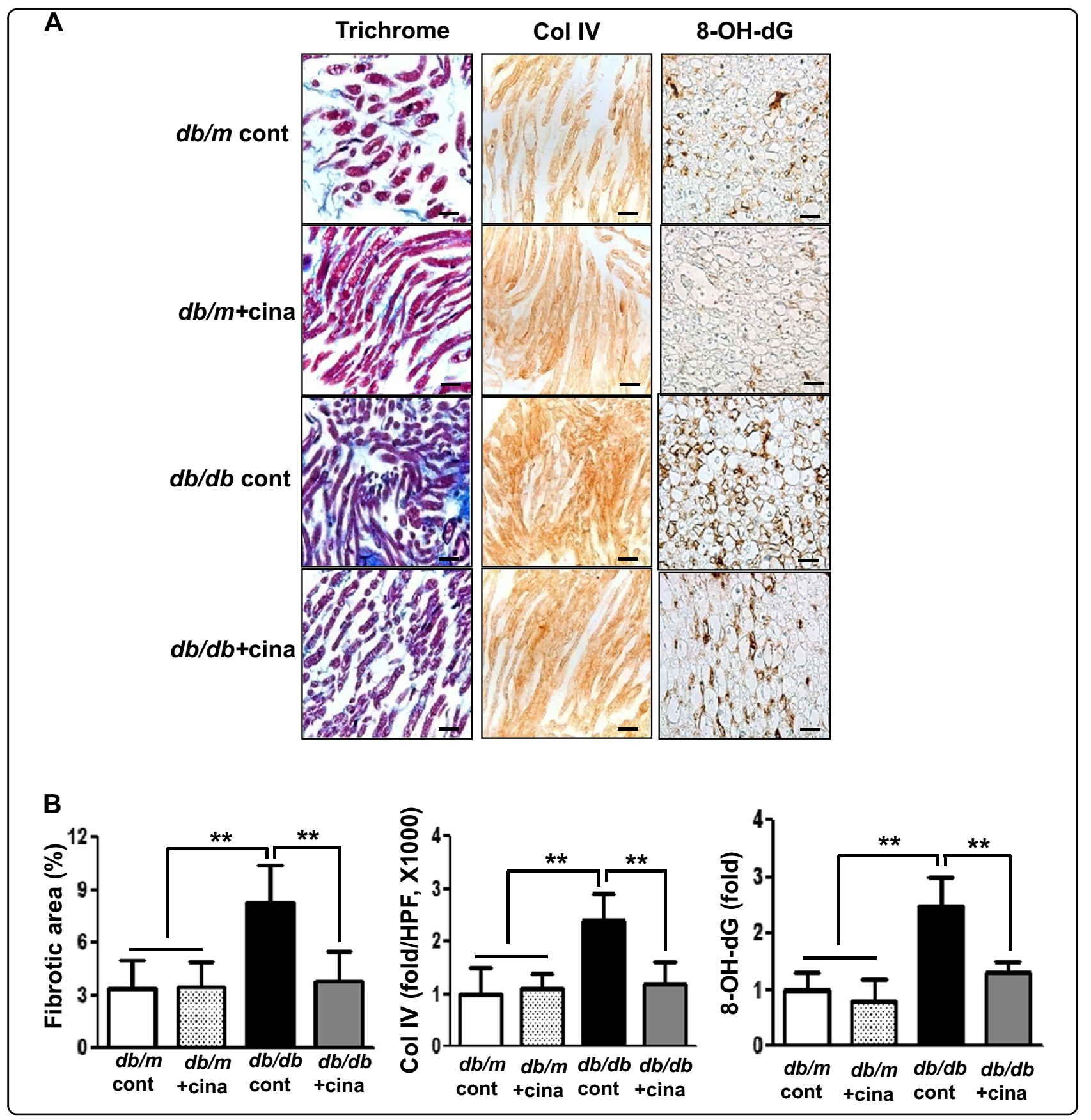



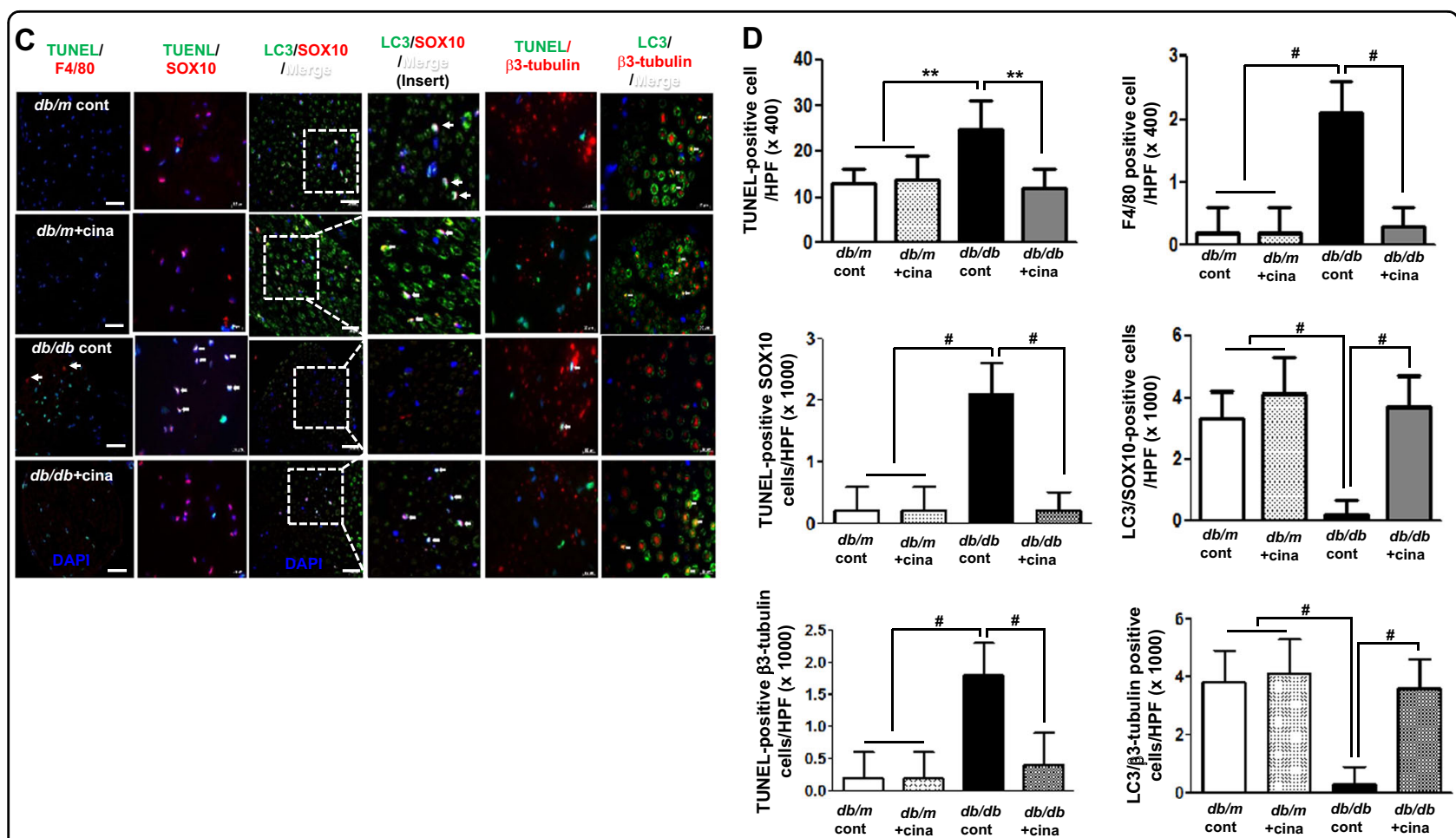

Fig. 2 Cinacalcet attenuates fibrosis, inflammation, and apoptosis of the sciatic nerve in $\mathbf{d b} / \mathbf{d} b$ mice. a-d. Nerve fibrosis, oxidative stress, inflammatory cell infiltration, and apoptosis in the sciatic nerves were determined at 20 weeks in $\mathrm{db} / \mathrm{m}$ and $\mathrm{db} / \mathrm{db}$ mice with or without cinacalcet treatment. Representative Masson's trichrome staining and immunohistochemical staining for Col IV (a, b) and 8-hydroxy-deoxyguanosine (8-OH-dG), immunofluorescence for TUNEL, F4/80-positive cells, TUNEL-SOX10- and TUNEL- $\beta 3$ tubulin-positive cells, and LC3-SOX10- and LC3- $\beta 3$ tubulinpositive cells were determined (b). The white dotted line box indicates the area for each enlarged figure. The white arrows indicate TUNEL-SOX10and TUNEL- $\beta 3$ tubulin-positive cells and LC3-SOX10- and LC3- $\beta 3$ tubulin-positive cells. The quantitative analyses of the results are shown (d, original magnification, $\times 1000)$. Scale bar $=10 \mu \mathrm{m}(\mathbf{a}, \mathbf{c})$. $\left(n=8\right.$ in each groups) ${ }^{* *} p<0.01$, and ${ }^{\#} p<0.001$ compared with the other groups

Expression levels of CaSR, CaMKK $\beta$, phospho-Ser ${ }^{428}$ and total LKB1, phospho-Thr ${ }^{172}$ and total AMPK expression,

PGC-1a, phospho-Ser ${ }^{1177}$ eNOS, Bcl-2, Bax, Beclin-1, and LC3-1 and -II in the sciatic nerve

On western blot analysis, CaSR, CaMKK $\beta$, phosphoLKB1, phospho-AMPK, PGC-1 $\alpha$, and phospho-eNOS expression was significantly decreased in the sciatic nerve of $d b / d b$ cont compared with that of $d b / m$ cont mice (Fig. 4a, b). These findings suggest that diabetes itself decreases the activation of CaSR-CaMKK $\beta$ and LKB1 phosphorylation, resulting in a decrease in AMPK phosphorylation, which seems to be related to the development of diabetic peripheral nerve damage. In contrast, cinacalcet treatment increased the expression of CaSR, CaMKK $\beta$, phospho-Ser ${ }^{428}$ LKB1, and phospho$\mathrm{Thr}^{172}$ AMPK, which subsequently recovered PGC- $1 \alpha$ and eNOS expression in the sciatic nerve of $d b / d b$ mice (Fig. 4a, b). For further evaluation of apoptosis and autophagy, we also measured the expression of Bcl-2/ Bax ratio, Beclin-1, and LC3-II/LC3-I ratio in the sciatic nerves. Significant decreases in the expression of Bcl-2/Bax, Beclin-1, and LC3-II/LC3-I ratio in the sciatic nerve of $d b / d b$ mice increased with cinacalcet treatment to the levels comparable to those of $\mathrm{db} / \mathrm{m}$ mice (Fig. 4c, d).

\section{Effects of cinacalcet on $\left[\mathrm{Ca}^{++}\right] \mathrm{i}$ in HSCs}

It is well known that an increase in $\left[\mathrm{Ca}^{++}\right] \mathrm{i}$ is associated with the activation of CaMKK and LKB1, which are potent activators of AMPK. Therefore, we measured the effect of cinacalcet on $\left[\mathrm{Ca}^{++}\right] \mathrm{i}$ in the HSCs grown in either low- or high-glucose medium with or without cinacalcet. Interestingly, both 15 and $100 \mathrm{nM}$ of cinacalcet significantly increased the peak $\left[\mathrm{Ca}^{++}\right] \mathrm{i}$ levels and its area under curve (Fig. 5a, b). We further evaluated the effects of cinacalcet on AMPK activation in cultured HSCs by immunofluorescence staining and western blot analysis. The intracellular LKB-1-AMPK-PGC- $1 \alpha$-eNOS signaling pathway is thought to play an important role in the maintenance of normal SC function. Thus we investigated the upstream signals of AMPK; significant decreases in CaSR, CaMKK $\beta$, phospho-Ser ${ }^{428}$ LKB1, and phospho$\mathrm{Thr}^{172}$ AMPK expression were noted in HSCs grown in high-glucose medium, resulting in subsequent decreases in PGC-1 $\alpha$ and phospho-Ser ${ }^{1177}$ eNOS expression, which are downstream targets of AMPK (Fig. 6a, b). 


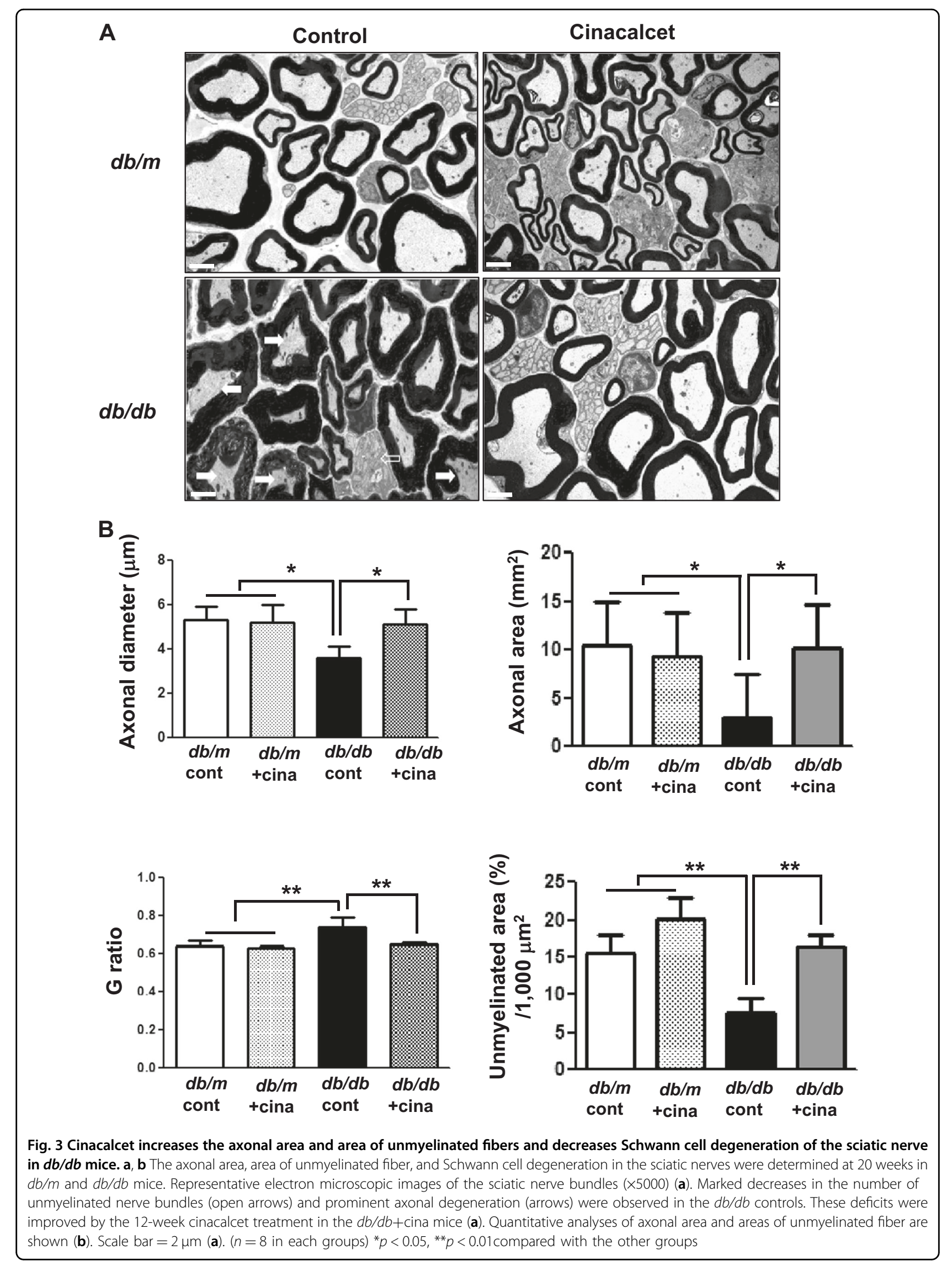




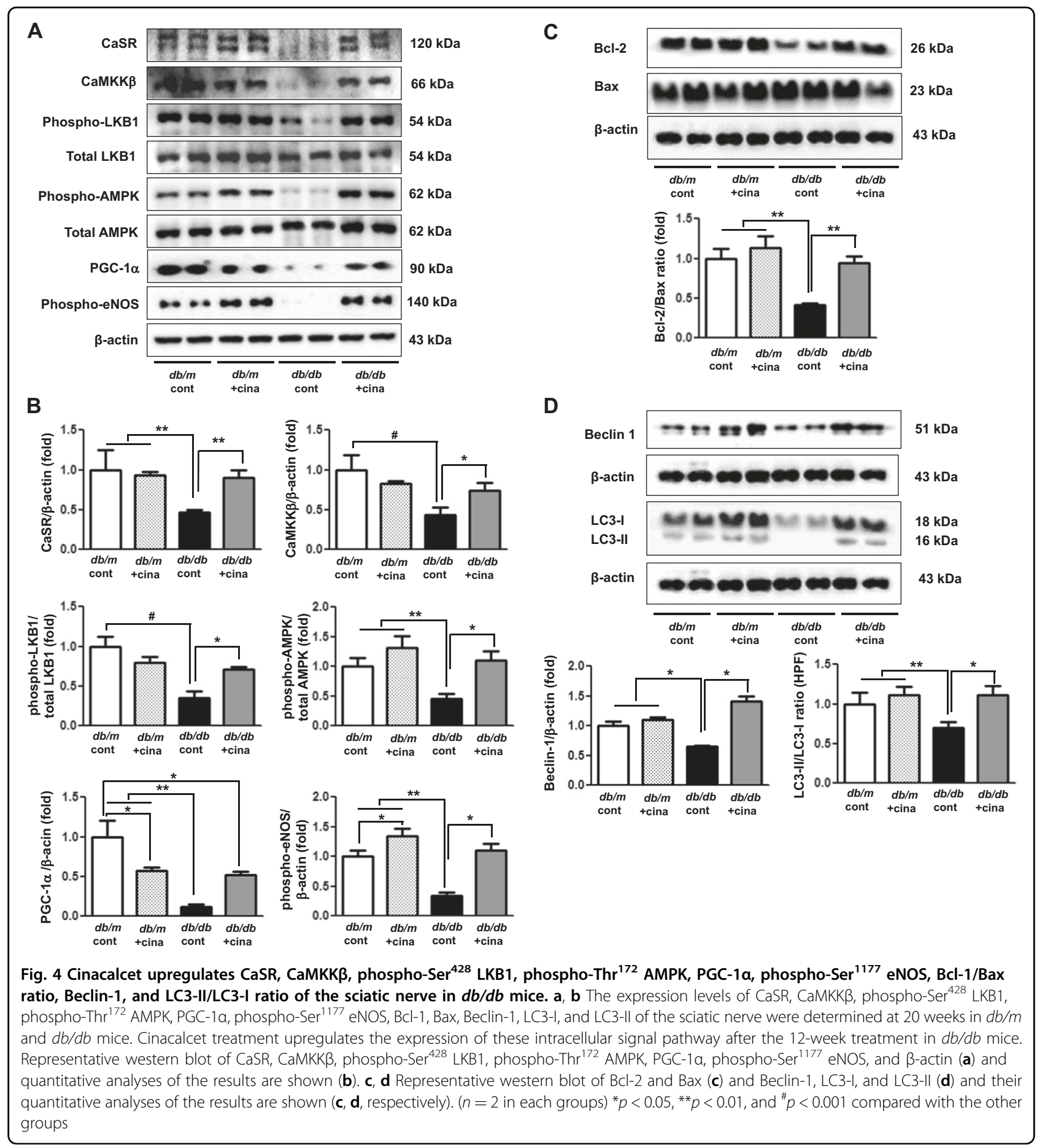

Consistent with the intracellular signaling changes, decreased extracellular NOx concentration and Bcl-2/Bax ratio in high-glucose medium was increased significantly with cinacalcet treatment with subsequent decrease in TUNEL-positive cells in high-glucose medium (Fig. 6b-d). Such changes were not observed either in low-glucose medium or in osmotic control (not shown), suggesting that high-glucose medium takes part in the suppression of $\left[\mathrm{Ca}^{++}\right] \mathrm{i}-\mathrm{CaMKK} \beta$-LKB-1-phospho-AMPK signaling and subsequent PGC- $1 \alpha$-phospho-eNOS-NOx axis in HSCs, which resulted in increased apoptotic cell deaths of HSCs. We also evaluated whether AMPK phosphorylation by cinacalcet preserves autophagy activity in SCs. The high-glucose-induced decreases in beclin1, LC3-II/LC3-I ratio, and number of LC3 punctate in SCs were completely recovered by cinacalcet treatment to the 

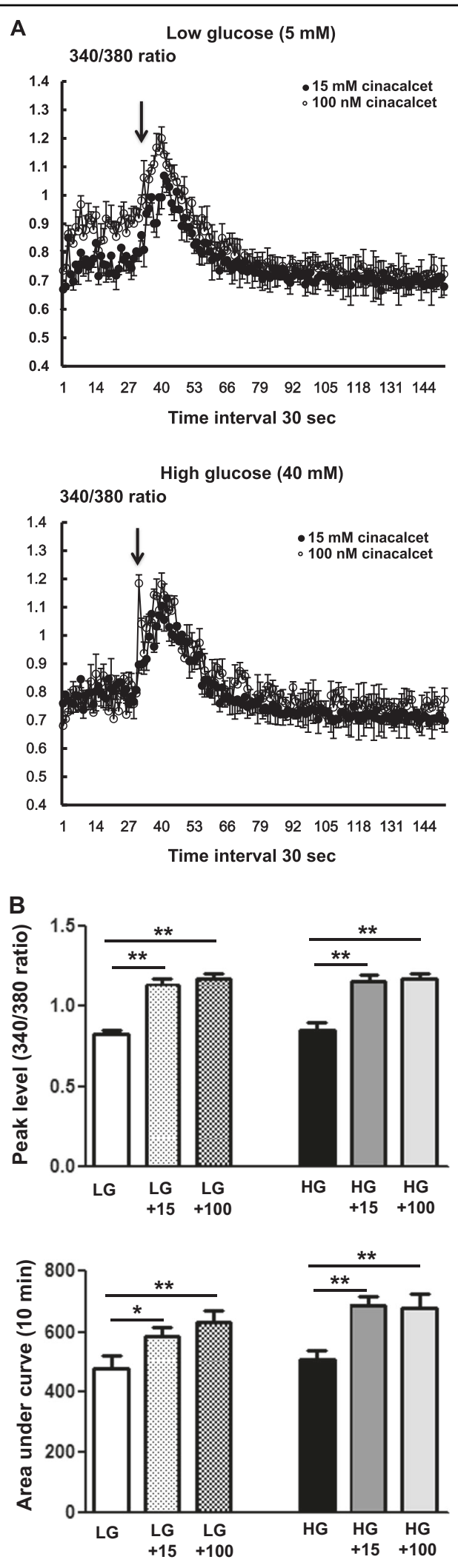

Fig. 5 Cinacalcet increases $\left[\mathrm{Ca}^{++}\right] \mathrm{i}$ in HSCs grown in high-glucose medium. $\mathbf{a}, \mathbf{b}$. To determine whether the addition of cinacalcet might modulate $\left[\mathrm{Ca}^{++}\right]$i in HSCs, FURA-2AM-loaded human Schwann cells (HSCs) were stimulated using different concentrations $(15,100 \mathrm{nM})$ of cinacalcet in low-glucose (LG; $5 \mathrm{mmol} / \mathrm{l}$ D-glucose) or high-glucose ( $\mathrm{HG} ; 40 \mathrm{mmol} / \mathrm{l}$ D-glucose) medium. The area under curve (AUC) was estimated from the baseline of normalized data (at the point of injection) to a fluorescence level and between time points of injection ( $0 \mathrm{~min}$ ) and $10 \mathrm{~min}$. The peak of the curve was measured as the highest value of the curve. The peak amplitude and $\mathrm{AUC}$ of $\left[\mathrm{Ca}^{++}\right] \mathrm{i}$ were significantly increased by cinacalcet in dose-dependent manners $(\mathbf{a}, \mathbf{b})$ in both $L G$ and $\mathrm{HG}$ media. In $\mathbf{a}$, the arrow denotes the administration of cinacalcet (15 and $100 \mathrm{nM}$, respectively). ( $n=6$ independent experiments in each experiments). ${ }^{*} p<0.05,{ }^{* *} p<0.01$, and ${ }^{\#} p<0.001$ compared with the other groups

levels similar to those of HSCs grown in low-glucose medium (Fig. 6e).

Transfection of HSCs with CaMKK $\beta$ and LKB1 siRNAs suppressed the expression of CaMKK $\beta$ and phospho$\operatorname{Ser}^{428}$ LKB1 by $80 \%$ and $60 \%$, respectively, in low-glucose medium (Fig. 7a). Transfection with either CaMKK $\beta$ or $L K B 1$ siRNA resulted in the dual suppression in the expression of CaMKK $\beta$ and phospho-LKB1 despite cinacalcet treatment. Moreover, Cinacalcet treatment did not increase the expression of phospho-Thr ${ }^{172}$ AMPK-PGC$1 \alpha$-phospho-Ser ${ }^{1177}$ eNOS-NOx signaling in HSCs when transfected with either CaMKK $\beta$ or LKB1 siRNA (Fig. 7b, c).

\section{Assessment of sciatic nerve function and phenotypes in 8- week-old $d b / m$ and $d b / d b$ mice}

To evaluate the effect of cinacalcet on the prevention and restoration of DPN, we investigated the functional and phenotypic changes in the sciatic nerves of 8-weekold $d b / m$ and $d b / d b$ mice before the treatment. While tactile threshold was significantly increased in the sciatic nerve of $d b / d b$ mice $(p<0.05)$, there were no significant differences in motor conduction latency and action potential amplitude between that of $d b / m$ and $d b / d b$ mice (Fig. 8a). However, area of fibrosis, oxidative stress, and neuronal degeneration including decreased axonal diameter and area were prominent in the sciatic nerve of $d b / d b$ mice with increased $G$ ratio and decreased area for unmyelinated fibers (Fig. 8b). Furthermore, increased expression of F4/80- and TUNELpositive cells and decreased expression of LC3-positive cells were noted in the sciatic nerve of $d b / d b$ mice compared with those in $d b / m$ mice (Fig. 8c) and these changes were in line with decreased expression of CaSRAMPK-eNOS signaling pathway in the same group (Fig. 8d). 


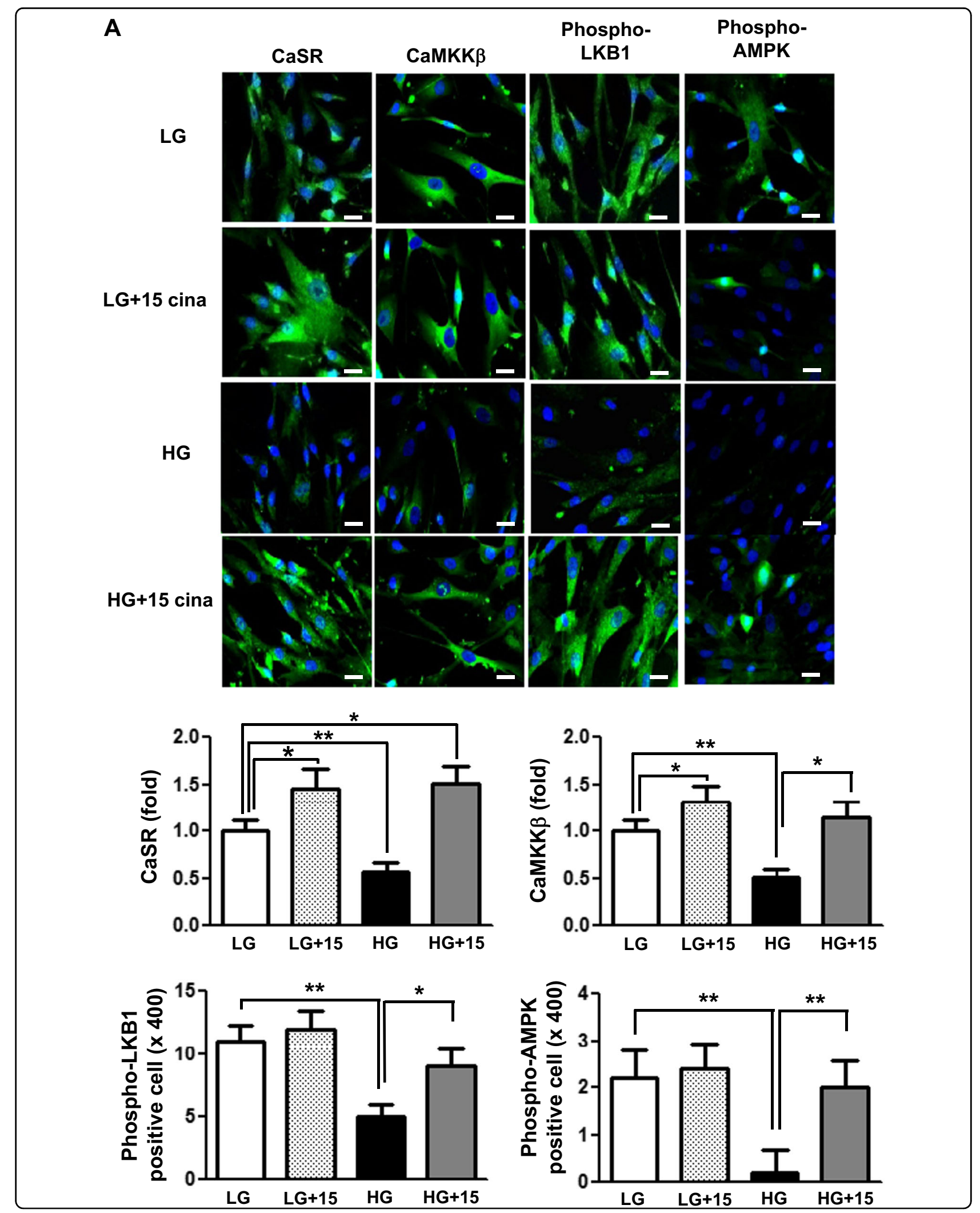




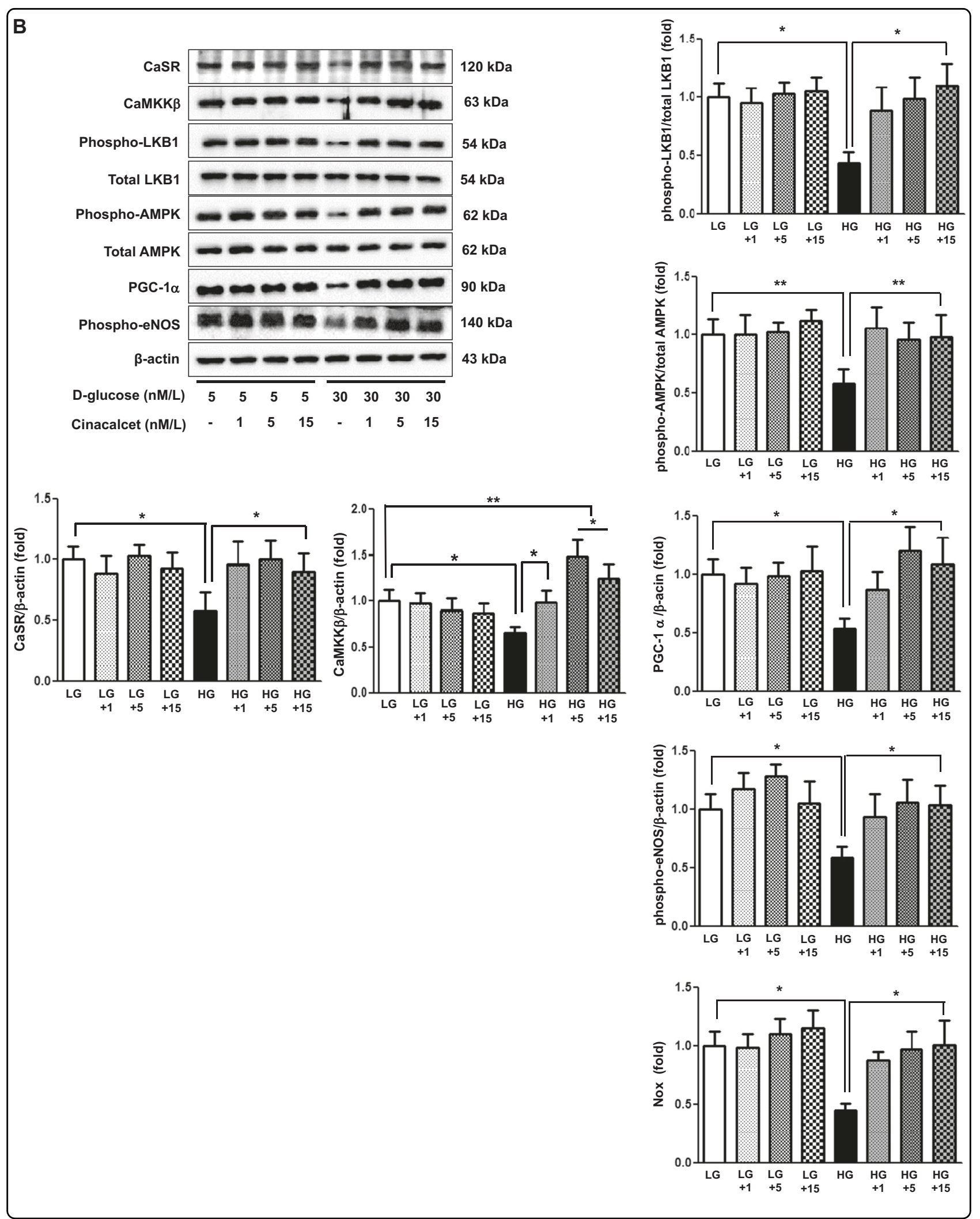




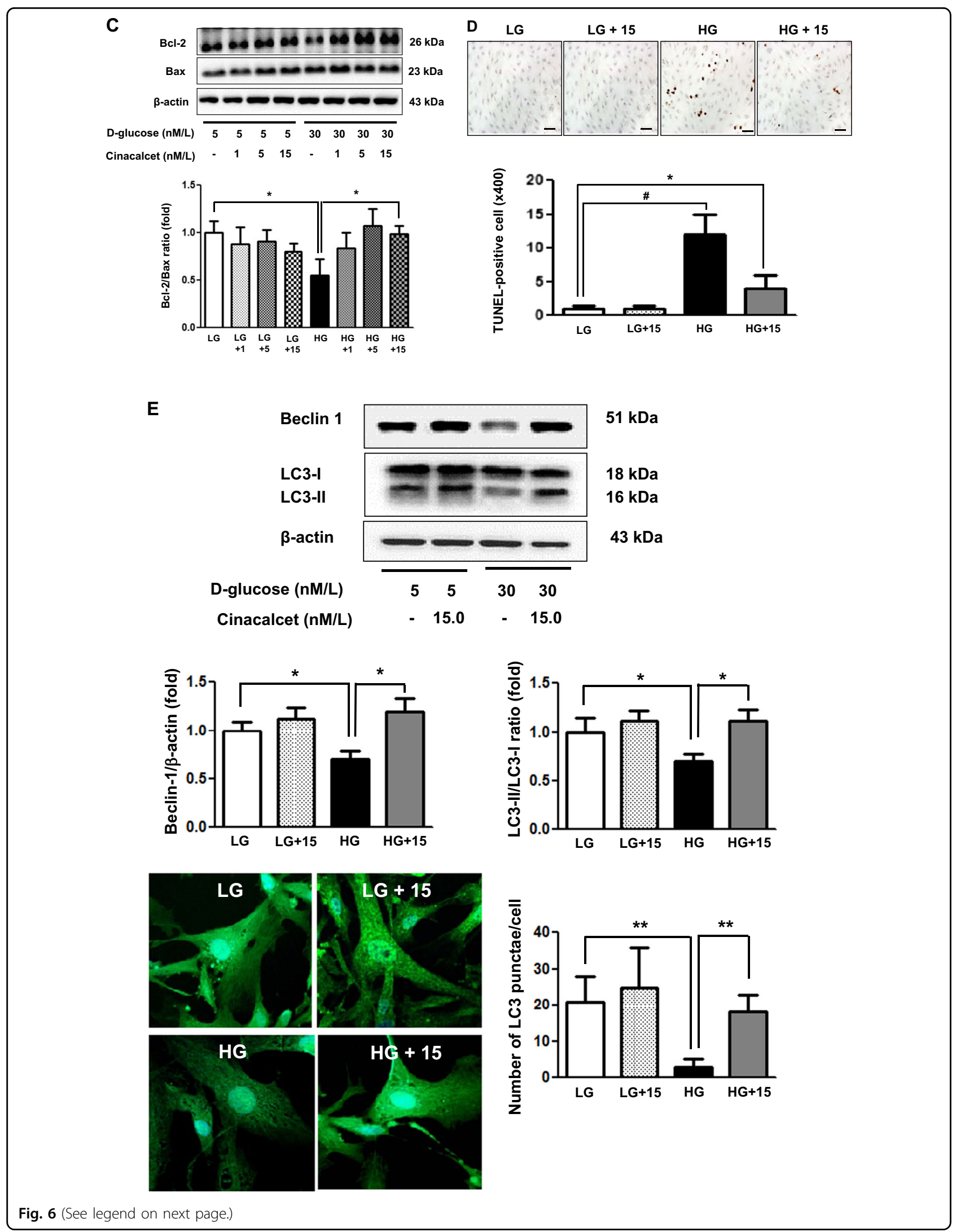


(see figure on previous page)

Fig. 6 Cinacalcet activates intracellular CaSR, CaMKK $\beta$, phospho-Ser ${ }^{428}$ LKB1, phospho-Thr ${ }^{172}$ AMPK, PGC-1a, phospho-Ser ${ }^{1177}$ eNOS, and NOx in HSCs grown in high-glucose medium, which prevents oxidative stress and apoptosis. a Immunofluorescence analysis was performed for CaSR, CaMKKB, and phosphor-Ser ${ }^{428}$ LKB1 in the HSCs with or without cinacalcet treatment (15 nM; original magnification, $\left.\times 400\right)$ and the quantitative analyses of the results are shown. Scale bars represent $30 \mu \mathrm{m}$. ( $n=6$ independent experiments in each experiments) (a). b, c The effect of cinacalcet on intracellular signals and apoptosis in the human Schwann cells (HSCs) cultured in low-glucose (LG; 5 mmol/l D-glucose) or highglucose (HG; $40 \mathrm{mmol} / \mathrm{l}$ D-glucose) conditions without or with dose-dependent cinacalcet treatment $(1,5,15 \mathrm{nM})$ were determined. CaSR, CaMKKa/ $\beta$, total LKB1, phosphor-Ser ${ }^{428}$ LKB1, total AMPK, phospho-Thr ${ }^{172}$ AMPK, PGC-1a, phospho-Ser ${ }^{1177}$ eNOS, NOx, BCL-2, BAX, and $\beta$-actin levels were assessed in the cultured HSCs. Representative western blot analyses and quantitative analyses of CaSR, CaMKKa/ $\beta$, total LKB1, phospho-Ser ${ }^{428}$ LKB1, total AMPK, phospho-Thr ${ }^{172}$ AMPK, PGC-1a, and phospho-Ser ${ }^{177}$ eNOS are shown (b). The NOx concentrations from the supernatant of HSCs are shown (b). Representative western blot analyses and quantitative analyses of BCL-2 and BAX are shown (c). d, e. Marked increases in TUNEL-positive HSCs were observed in the HG medium compared to HG+15 group. The quantitative analyses of the results are shown (d). Representative western blot analyses and quantitative analyses of Beclin-1, LC3-1, and LC3-II are shown (e). The quantitative analyses of the results are shown (e). Marked decreases in the number of punctate in an SC were observed in the HG medium compared to HG+15 group (e). ( $n=4$ independent experiments in each experiments) ${ }^{*} p<0.05,{ }^{* *} p<0.01$, and ${ }^{\#} p<0.001$ compared with the other groups

\section{Discussion}

The current study provides empirical evidences that cinacalcet improved sensorimotor function and restored damaged nerve phenotypes including nerve fibrosis and inflammation, axonal degeneration, loss of unmyelinated fibers, and apoptotic neuronal cell loss in the sciatic nerve of diabetic mice. Along with these changes, cinacalcet also restored defective autophagy activity in both SCs and peripheral nerve, which characterizes early-stage diabetic neuropathy. Activation of CaMKK $\beta$ and phosphorylation of LKB1 by cinacalcet increased phosphorylation of AMPK that subsequently activated PGC-1 $\alpha$ and phospho$\mathrm{Ser}^{1177}$ eNOS-NO and increased the ratio of $\mathrm{Bcl}-2 / \mathrm{Bax}$, beclin-1, and LC3-II/LC3-I.

It is established that impaired $\left[\mathrm{Ca}^{++}\right] \mathrm{i}$ homeostasis is implicated in the development of $\mathrm{DPN}^{25,26}$. Previous studies supported the notion that deranged $\mathrm{Ca}^{++}$homeostasis is attributable to impaired sarco/endoplasmic reticulum calcium ATPase pumps located in the endoplasmic reticulum membrane ${ }^{19}$. Therapy with low-dose insulin and neurotrophin-3 restored resting $\mathrm{Ca}^{++}$levels from intracellular stores, signifying that altered calcium homeostasis could be an early molecular marker linked to the onset of diabetic sensory neuropathy ${ }^{27,28}$. In the current study, cinacalcet treatment increased the expression of $\mathrm{CaSR}$ and $\left[\mathrm{Ca}^{++}\right] \mathrm{i}$ in cytoplasm in association with subsequent increase in the expression of CaMKK $\beta$ and LKB-1 in the diabetic animals and cultured SCs that was independent of adenylate energy balance, such as AMP/ ATP and ADP/ATP ratios. More importantly, transfection with either $C a M K K \beta$ or $L K B 1$ siRNA resulted in the dual suppression in the expression of both CaMKK $\beta$ and LKB1 and their downstream phospho-Thr ${ }^{172}$ AMPK, PGC-1 $\alpha$, and phosphor-Ser ${ }^{1177}$ eNOS signaling in HSCs cultured in high-glucose medium. The results signify the dual activation of CaMKK $\beta$ and LKB1 as a prerequisite and that the interaction between the two upstream kinases is required for further enhancement of their downstream effectors by cinacalcet treatment.
AMPK is a major downstream effector of its upstream kinases that plays a key role in cell survival and death in response to metabolic stress. The role of AMPK activation in restoring nerve function, preventing, and even reversing pathological pain associated with DPN is implicated in various studies; we previously demonstrated that fenofibrate treatment ameliorated neuronal damage in the sciatic nerve of type 2 diabetic mice by activating the PPAR $\alpha$-AMPK-PGC-1 $\alpha$-eNOS pathway ${ }^{15}$. Moreover, mitochondrial dysfunction in DPN is characterized by maladaptation in such metabolic signaling pathway as AMPK/sirtuin-PGC- $1 \alpha$ axis that contributes to the diminishment of axonal regeneration capacity ${ }^{29}$. In line with this, diabetes-associated alterations in the peripheral nerve phenotype and concomitant development of sensorimotor dysfunction were accompanied by decreased expression of AMPK-PGC- $1 \alpha$-eNOS signaling and this was ameliorated by cinacalcet treatment through the upregulation of AMPK-eNOS phosphorylation in the sciatic nerve of diabetic mice.

One favorable and potential downstream mediator of AMPK signaling is $\mathrm{NO}^{30}$. Neuronal damages may be associated with secondary deficits in endothelial function resulting from impaired $\mathrm{NO}$ synthesis, release of $\mathrm{NO}$ upon endothelial injury by oxidative stress, and increased free radical activity. Cinacalcet's favorable effects on preventing sensorimotor dysfunction and neuronal damage may be implemented by AMPK-induced modulation of eNOS and enhancement of NOx production, which preserves peripheral nerve, especially SCs, and promotes endothelial cell survival and function.

To explore the cellular fate of peripheral nerves associated with enhanced eNOS-NOx activation, we determined the degree of autophagic activity as represented by LC3-II/LC3-I. LC3-II serves as a molecular biomarker for the assessment of autophagic activity. Impaired autophagic activity participates in the development of a variety of disease including neurodegenerative disorders probably due to the accumulation of damaged molecules 


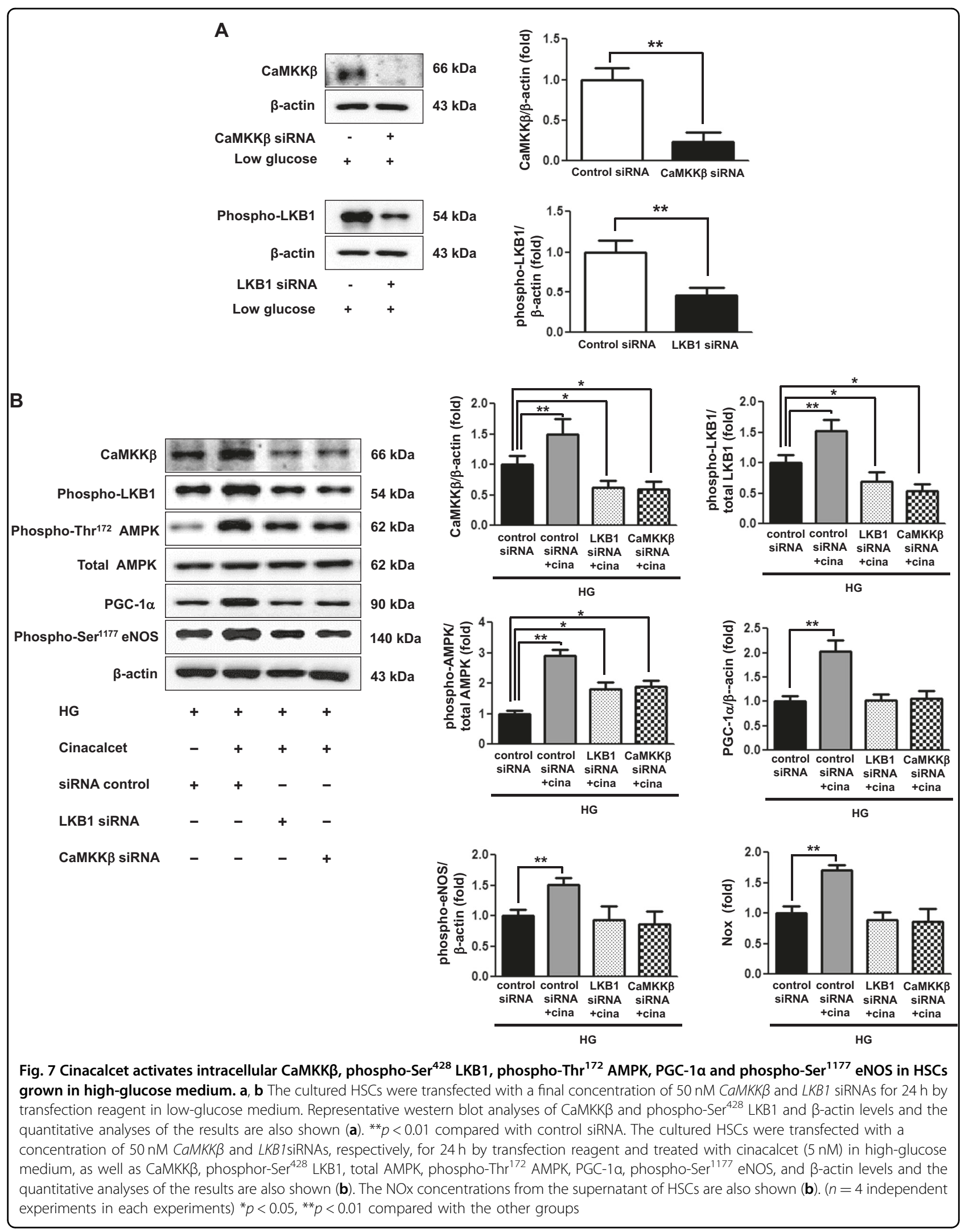




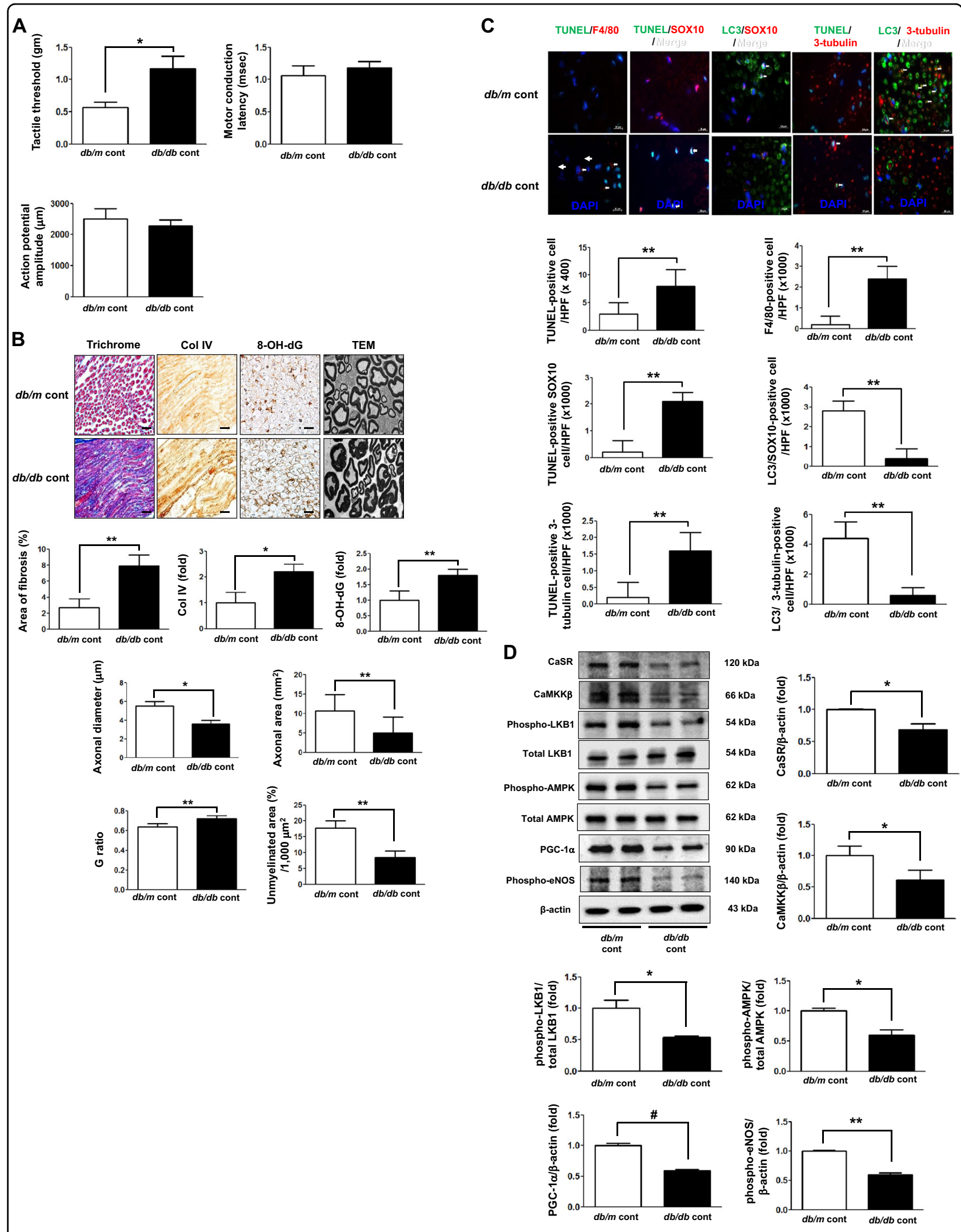

Fig. 8 (See legend on next page.) 
(see figure on previous page)

Fig. 8 Functional and phenotypic changes in the sciatic nerve of 8 -week-old $d \boldsymbol{d} / \mathbf{m}$ and $\boldsymbol{d} \boldsymbol{b} \mathbf{b} \mathbf{d} \boldsymbol{b}$ mice. a Effects of cinacalcet on the tactile threshold, motor conduction latency, and action potential amplitude were determined. b Nerve fibrosis (Masson's trichrome and Col IV), oxidative stress $(8-\mathrm{OH}-\mathrm{dG})$, and the axonal diameter and area, the $\mathrm{G}$ ratio, and area of unmyelinated fiber in the sciatic nerves were determined. Representative electron microscopic images of the sciatic nerve bundles $(\times 5000)$ are shown. Scale bars represent $2 \mu \mathrm{m}$. c Immunofluorescences for TUNEL, F4/80positive cells, TUNEL-SOX10- and TUNEL- $\beta 3$ tubulin-positive cells, and LC3-SOX10- and LC3- $\beta 3$ tubulin-positive cells were determined. The white arrows indicate TUNEL-SOX10- and TUNEL- $\beta 3$ tubulin-positive cells and LC3-SOX10- and LC3- $\beta 3$-tubulin-positive cells, respectively. The quantitative analyses of the results are shown (c, original magnification, $\times 1000)$. Scale bars represent $10 \mu \mathrm{m}(\mathbf{b}, \mathbf{c})$. $(n=6$ independent experiments in each experiments) ${ }^{*} p<0.05$ and ${ }^{* *} p<0.01$ compared with the $d b / m$ cont group. $\mathbf{d}$ The expression levels of CaSR, CaMKK $\beta$, phospho-Ser ${ }^{428}$ LKB1, phospho$\mathrm{Thr}^{172}$ AMPK, PGC-1a, phospho-Ser ${ }^{1177}$ eNOS, BCl-1, and $\beta$-actin of the sciatic nerve were determined. Representative western blot of CaSR, CaMKK $\beta$, phospho-Ser ${ }^{428}$ LKB1, phospho-Thr ${ }^{172}$ AMPK, PGC-1a, phospho-Ser ${ }^{1177}$ eNOS, and $\beta$-actin and quantitative analyses of the results are shown (d). $(n=$ 4 independent experiments in each experiments) ${ }^{*} p<0.05,{ }^{* *} p<0.01$, and ${ }^{*} p<0.001$ compared with the other groups

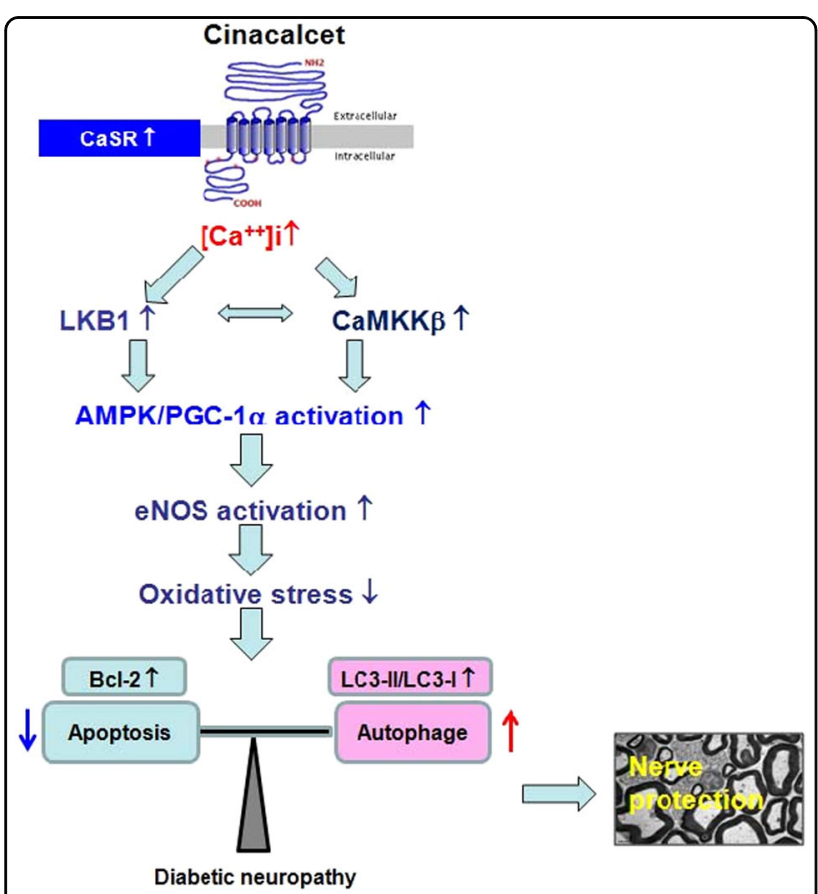

Fig. 9 The proposed role of cinacalcet in diabetic peripheral neuropathy and the interplay between cinacalcet and peripheral nerve injury in type 2 diabetes. AMPK AMP-activated protein kinase, CaMKK $\mathrm{Ca}^{2+} /$ calmodulin-dependent protein kinase kinase- $\beta$, eNOS endothelial nitric oxide synthase, LKB1 liver kinase B1, $\left[\mathrm{Ca}^{++}\right] \mathrm{i}$ intracellular $\mathrm{Ca}^{++}$

and organelles that promotes cell death. On the other hand, excessive autophagy could also result in cell death and dysfunction by facilitating apoptosis with potential clinical significance, and therefore, the proportional contribution of autophagy ${ }^{31}$ and apoptosis and the balance between the two counteracting functions is essential in promoting cell viability and further maintaining the functional and phenotypic integrity of peripheral nerves in diabetes. In this aspect, cinacalcet may exhibit its potential as a means to promote cell survival by enhancing autophagy and attenuating apoptosis.
In summary, this study strongly suggests a favorable effect of cinacalcet in DPN by enhancing the $\left[\mathrm{Ca}^{++}\right] \mathrm{i}-\mathrm{CaMKK} \beta$ LKB1 pathway and its downstream effectors AMPK-PGC$1 \alpha$-eNOS, which may prevent the sciatic nerve injury from diabetes-induced oxidative stress through not only decreased apoptosis but also increased autophagy activity (Fig. 9). Therefore, cinacalcet-induced AMPK activation underscores the modulation of autophagy and apoptosis in the sciatic nerve and may be a promising therapeutic means to deter and prevent the progression of DPN.

\section{Acknowledgements \\ This study was supported by grants from the Basic Science Research Program through the National Research Foundation of Korea (NRF) funded by the Ministry of Education, Science and Technology (H.W.K.: NRF- 2016R1A2B4015878; J.H.L.: NRF-2018R1D1A1B07048315, C.W.P.: NRF- 2016R1A2B2015980), and the Seoul St. Mary's Hospital R\&D Project, The Catholic University of Korea (C.W.P.: 52015B000100004). This study was supported by the Young Investigator Research Grant from the Korean Society of Nephrology (J.H.L.; GAMBRO 2014). The authors wish to acknowledge the financial support of the St. Vincent's Hospital, Research Institute of Medical Science (H.W.K.: SVHR-2014-04) and the Catholic Medical Center Research Foundation made in the program year of 2012.}

\section{Author details}

'Department of Rehabilitation Medicine, College of Medicine, The Catholic University of Korea, Seoul, Korea. ${ }^{2}$ Division of Nephrology, Department of Internal Medicine, College of Medicine, The Catholic University of Korea, Seoul, Korea. ${ }^{3}$ Institute for Aging and Metabolic Diseases, College of Medicine, The Catholic University of Korea, Seoul, Korea

\section{Authors' contributions}

Y.C.C. and J.H.L. wrote the manuscript and researched data. H.M.O., H.W.K., M.Y.K., E.N.K. and Y.K. performed the study, analyzed data, and contributed to the discussion. Y.S.C. contributed to discussion and reviewed. H.W.K. and C.W.P. researched data, contributed to discussion, and edited the manuscript.

\section{Conflict of interest}

The authors declare that they have no conflict of interest.

\section{Publisher's note}

Springer Nature remains neutral with regard to jurisdictional claims in published maps and institutional affiliations.

Received: 7 June 2018 Revised: 10 October 2018 Accepted: 11 October 2018

Published online: 26 November 2018 


\section{References}

1. Sima, A. A., Thomas, P. K., Ishii, D. \& Vinik, A. Diabetic neuropathies. Diabetologia 40(Suppl 3), B74-B77 (1997).

2. Greene, D. A., Stevens, M. J. \& Feldman, E. L. Diabetic neuropathy: scope of the syndrome. Am. J. Med. 107, 2s-8s (1999).

3. The Diabetes Control and Complications Trial Research Group. The effect of intensive treatment of diabetes on the development and progression of longterm complications in insulin-dependent diabetes mellitus. N. Engl. J. Med. 329, 977-986 (1993).

4. Cameron, N. E., Eaton, S. E., Cotter, M. A. \& Tesfaye, S. Vascular factors and metabolic interactions in the pathogenesis of diabetic neuropathy. Diabetologia 44, 1973-1988 (2001).

5. van Dam, P. S. Oxidative stress and diabetic neuropathy: pathophysiological mechanisms and treatment perspectives. Diabetes Metab. Res. Rev. 18, 176-184 (2002)

6. Anand, P. et al. The role of endogenous nerve growth factor in human diabetic neuropathy. Nat. Med. 2, 703-707 (1996).

7. Yerra, V. G., Gundu, C., Bachewal, P. \& Kumar, A. Autophagy: the missing link in diabetic neuropathy? Med. Hypotheses 86, 120-128 (2016).

8. Simmons, Z. \& Feldman, E. L. Update on diabetic neuropathy. Curr. Opin Neurol. 15, 595-603 (2002).

9. Tomlinson, D. R. \& Gardiner, N. J. Glucose neurotoxicity. Nat. Rev. Neurosci. 9, 36-45 (2008).

10. Eckersley, L. Role of the Schwann cell in diabetic neuropathy. Int. Rev. Neurobiol. 50, 293-321 (2002).

11. Gonzalez, C. D. et al. The emerging role of autophagy in the pathophysiology of diabetes mellitus. Autophagy 7, 2-11 (2011).

12. $Y u, Y$. et al. Protective effect of hydrogen-rich medium against high glucoseinduced apoptosis of Schwann cells in vitro. Mol. Med. Rep. 12, 3986-3992 (2015).

13. Hardie, D. G., Hawley, S. A. \& Scott, J. W. AMP-activated protein kinase-development of the energy sensor concept. J. Physiol. 574, 7-15 (2006).

14. Chowdhury, S. K., Dobrowsky, R. T. \& Fernyhough, P. Nutrient excess and altered mitochondrial proteome and function contribute to neurodegeneration in diabetes. Mitochondrion 11, 845-854 (2011).

15. Cho, Y. R. et al. Therapeutic effects of fenofibrate on diabetic peripheral neuropathy by improving endothelial and neural survival in $\mathrm{db} / \mathrm{db}$ mice. PLoS ONE 9, e83204 (2014).

16. Shaw, R. J. et al. The tumor suppressor LKB1 kinase directly activates AMPactivated kinase and regulates apoptosis in response to energy stress. Proc. Natl Acad. Sci. USA 101, 3329-3335 (2004).

17. Jensen, T. E. et al. Possible CaMKK-dependent regulation of AMPK phosphorylation and glucose uptake at the onset of mild tetanic skeletal muscle contraction. Am. J. Physiol. Endocrinol. Metab. 292, E1308-E1317 (2007).

18. Brown, E. M. \& MacLeod, R. J. Extracellular calcium sensing and extracellular calcium signaling. Physiol. Rev. 81, 239-297 (2001).

19. Bukoski, R. D., Bian, K., Wang, Y. \& Mupanomunda, M. Perivascular sensory nerve $\mathrm{Ca} 2+$ receptor and $\mathrm{Ca} 2+$-induced relaxation of isolated arteries. Hypertension 30, 1431-1439 (1997).

20. Beirowski, B. et al. Metabolic regulator LKB1 is crucial for Schwann cellmediated axon maintenance. Nat. Neurosci. 17, 1351-1361 (2014).

21. Verkhratsky, A. \& Fernyhough, P. Calcium signalling in sensory neurones and peripheral glia in the context of diabetic neuropathies. Cell Calcium 56, 362-371 (2014).

22. Dasgupta, B. \& Milbrandt, J. Resveratrol stimulates AMP kinase activity in neurons. Proc. Natl Acad. Sci. USA 104, 7217-7222 (2007).

23. Sharma, S. S., Kumar, A., Arora, M. \& Kaundal, R. K. Neuroprotective potential of combination of resveratrol and 4-amino 1,8 naphthalimide in experimental diabetic neuropathy: focus on functional, sensorimotor and biochemical changes. Free Radic. Res. 43, 400-408 (2009).

24. Rushton, W. A. A theory of the effects of fibre size in medullated nerve. J. Physiol. 115, 101-122 (1951).

25. Khomula, E. V. et al. Specific functioning of Cav3.2 T-type calcium and TRPV1 channels under different types of STZ-diabetic neuropathy. Biochim. Biophys. Acta 1832, 636-649 (2013).

26. Zherebitskaya, E. et al. Sensory neurons derived from diabetic rats have diminished internal Ca2+stores linked to impaired re-uptake by the endoplasmic reticulum. ASN Neuro 4, https://doi.org/10.1042/an20110038 (2012).

27. Burdakov, D., Petersen, O. H. \& Verkhratsky, A. Intraluminal calcium as a primary regulator of endoplasmic reticulum function. Cell Calcium 38, 303-310 (2005).

28. Huang, T. J., Sayers, N. M., Fernyhough, P. \& Verkhratsky, A. Diabetes-induced alterations in calcium homeostasis in sensory neurones of streptozotocindiabetic rats are restricted to lumbar ganglia and are prevented by neurotrophin-3. Diabetologia 45, 560-570 (2002).

29. Fernyhough, P. Mitochondrial dysfunction in diabetic neuropathy: a series of unfortunate metabolic events. Curr. Diab. Rep. 15, 89 (2015).

30. Li, J. et al. Role of the nitric oxide pathway in AMPK-mediated glucose uptake and GLUT4 translocation in heart muscle. Am. J. Physiol. Endocrinol. Metab. 287, E834-E841 (2004).

31. Kotiadis, V. N., Duchen, M. R. \& Osellame, L. D. Mitochondrial quality control and communications with the nucleus are important in maintaining mitochondrial function and cell health. Biochim. Biophys. Acta 1840, 1254-1265 (2014). 